\title{
Electrical storm: mechanistic and therapeutic considerations to avoid death in the survivors
}

\author{
Yukiomi Tsuji ${ }^{1}$, Dobromir Dobrev ${ }^{2}$ \\ ${ }^{1}$ Department of Molecular Physiology, Nagasaki University Graduate School of Biomedical Sciences, Nagasaki, Japan; ${ }^{2}$ Institute of Pharmacology, \\ West German Heart and Vascular Center, University of Duisburg-Essen, Essen, Germany \\ Correspondence to: Yukiomi Tsuji. 1-12-4 Sakamoto, Nagasaki 852-8523, Japan. Email: yukiomitsuji@nagasaki-u.ac.jp. \\ Provenance: This is an invited Editorial commissioned by the Section Editor Fang-Zhou Liu (Guangdong Cardiovascular Institute, Guangzhou, \\ China). \\ Comment on: Chatzidou S, Kontogiannis C, Tsilimigras DI, et al. Propranolol Versus Metoprolol for Treatment of Electrical Storm in Patients With \\ Implantable Cardioverter-Defibrillator. J Am Coll Cardiol 2018;71:1897-906.
}

Submitted Sep 08, 2018. Accepted for publication Sep 19, 2018.

doi: $10.21037 /$ jtd.2018.09.111

View this article at: http://dx.doi.org/10.21037/jtd.2018.09.111

Electrical storm (ES), defined as 3 or more ventricular tachycardia or fibrillation (VT/VF) episodes within a 24-h period, is a major clinical problem with substantial morbidity and mortality in implantable cardioverterdefibrillator (ICD) patients with heart failure (HF) and reduced left ventricular ejection fraction. The currently used therapy for ES is empiric, typically involving suppression with antiarrhythmic agents (e.g., amiodarone, sotalol and $\beta$-blockers), catheter ablation or a combination of both. However, the therapeutic and particularly prognostic value of these treatment options needs to be determined. The article by Chatzidou et al. published in $7 \mathrm{Am}$ Coll Cardiol 2018 is the first double-blind randomized controlled trial (RCT), in which 60 ICD patients with ES developed within $24 \mathrm{~h}$ from admission were enrolled and the efficacy of amiodarone combined with a non-selective versus a selective $\beta$-blocker was compared (1). Non-selective $\beta$-adrenoceptor blockade with propranolol was more efficient and effective at an earlier time point than selective $\beta_{1}$-adrenoceptor blockade with metoprolol for acute suppression of ES. The study demonstrates not only that amiodarone plus $\beta$-blocker therapy is the cornerstone pharmacological treatment of ES, but also provides important information on $\beta$-blocker selection in the management of ES. The possible mechanisms by which the $\beta_{2}$-adrenoceptor blockade with propranolol might contribute to the better outcome were discussed by Chen and Doytchinova in an accompanying editorial in the same issue of $7 \mathrm{Am}$ Coll Cardiol (2).

While ICDs can be life-saving, ICD shocks are associated with an increase in mortality and with worsening HF. ES patients receiving multiple shocks for repeated VT/VF have more serious consequences than those with isolated VT/ $\mathrm{VF}$ unrelated to ES. It remains unclear, however, whether shocks play a causal role or whether this correlation is due solely to the underlying disease. Chatzidou et al. showed that ICD shocks were significantly lower in the propranolol group during an observation period of $48 \mathrm{~h}$ (1). It would be important to investigate whether the early termination of ES and shock reduction by propranolol plus amiodarone impacts the outcome beyond the acute period. In this editorial, we discuss the aspects of shock-associated mortality by reviewing the recently-published clinical and experimental studies and consider the possibility of a mechanism-based therapeutic strategy to reduce the risk of death.

\section{Currently used therapies and mortality}

Modern ICD programming to treat VT without shocks and to avoid unnecessary shocks and recent advance in mapping technologies and catheter ablation techniques to treat VT lead to a substantial reduction in inappropriate and/or appropriate ICD interventions, but this did not in a consistent mortality benefit. Programming with longer detection intervals and/or higher detection rates results in a large reduction in mortality in patients with ICDs for primary prevention (3), but is less beneficial for patients who already had VT/VF $(4,5)$. Prognostic advantage of 
catheter ablation was reported in a large-scaled RCT VANISH study (6), in which there was a clear difference in the composite primary outcome of death, VT storm, or appropriate ICD shock between the catheter ablation group and the escalation of antiarrhythmic drugs therapy group. Nevertheless, the Kaplan-Meier curve of death rate was almost identical between the two groups, suggesting that the superiority of catheter ablation is rather minimal. Moreover, a meta-analysis of RCTs to compare effectiveness of antiarrhythmic drugs versus catheter ablation for preventing VT in ICD patients showed that although a significant reduction in appropriate ICD shocks for VT/VF was comparable, neither antiarrhythmic drugs nor catheter ablation was associated with a decrease in mortality (7). These clinical findings suggest that shock reduction is not necessarily connected to a mortality benefit and thus the adverse outcome after ICD shocks is more closely related to the natural progression of the failing heart rather than to the harmful effects of the shocks. Also, optimization of HF regimes with proven mortality-protection is recommended in ICD patients, especially with ES. However, the high prevalence of $\beta$-blockers, angiotensin convertingenzyme inhibitors or angiotensin-II-receptor blockers, mineralocorticoid-receptor antagonists and loop diuretics in ES patients does not alter the poor outcomes (8).

\section{Mechanistic and therapeutic considerations}

Clearly, development of novel therapeutic approaches is required and desirable to reduce the risk of death associated with shocked VT/VF, but lack of understanding of the underlying mechanisms strongly limits the success of ICD patient management.

Electrical shocks-induced cardiac damage is considered as a strong contributor to poor outcomes. Defibrillation shocks transiently deteriorate the heart, causing mild elevation of serum cardiac troponin-I and a decrease in the myocardial lactate extraction rate by mitochondria, along with pathological and ultrastructural changes. Many of these alterations likely result from the disruption of cell membranes by electroporation due to the electrical shocks (9). This phenomenon is reversible within seconds in general, but there is experimental evidence that a single clinically relevant defibrillation shock induces electroporation with delayed recovery of membrane integrity at a region near the right ventricular (RV) electrode, the area $4 \%$ of the whole ventricles (10). However, the cellular consequences of electroporation, the extent and spatial distribution of irreversible electroporation that may have long-term adverse effects and the effects of exposure to multiple shocks remain unknown. In addition, whether shocks induce any electroporation of mitochondria and sarcoplasmic reticulum (SR) is also unclear.

An ICD shock apparently activates the sympathetic nervous system which is reflected by the transient $(\sim 10$ minutes) three-fold increase in systemic catecholamine levels just after an ICD shock for induced VF (11). Whether adrenergic emergence has long-term adverse effects to the heart is unknown. The occurrence of ICD shocks is associated with reductions in mental well-being and physical function and increases in depression and anxiety, the disorders that might be associated with chronic sympathetic activation (12).

The global ischemia during VF causes myocardial depression after defibrillation. Reactive oxygen species (ROS) formation and cytosolic $\mathrm{Ca}^{2+}$-overload which cause cardiac stunning due to ischemia/reperfusion likely also contribute to post-defibrillation cardiac stunning (13). However, electromechanical dissociation, the most serious complication in defibrillation testing in the clinical practice, often occurs in anesthetized patients with oxygenation. These observations suggest that excess cytosolic $\mathrm{Ca}^{2+}$ during VF may have important consequences for contractile function, independent of ischemia/reperfusion.

The potential mechanisms of shocked VT/VFassociated worsening HF are illustrated in Figure 1. Mitochondrial dysregulation, abnormal $\mathrm{Ca}^{2+}$-handling (SR ryanodine receptor dysfunction and SR ATPase $\mathrm{Ca}^{2+}$-pump depression) and cytosolic $\mathrm{Ca}^{2+}$-overload are hallmarks of the pathophysiology of HF. There is experimental and clinical evidence that defibrillation shocks cause structural and functional changes in mitochondria (14-16) and functional depression of SR Ca ${ }^{2+}$-pump (17). Serum levels of sFas, a biomarker of apoptosis, were highly elevated $24 \mathrm{~h}$ after defibrillation test in some patients at ICD implantation (14). Mitochondrial swelling, loss of membrane integrity, and mitochondrial crest disruption were observed in dogs with multiple low energy endocardial countershocks (15). Impaired complex activities and respiration and ROS were detected in rats with VF cardiac arrest resuscitation (16). Excess ROS directly leads to redox modification of ryanodine receptor and $\mathrm{SR} \mathrm{Ca}^{2+}$-pump, causing an increase in diastolic $\mathrm{Ca}^{2+}$-leak and a decrease in the $\mathrm{Ca}^{2+}$-uptake (18). The resulting cytosolic $\mathrm{Ca}^{2+}$-overload causes mitochondrial $\mathrm{Ca}^{2+}$-dysregulation (19). Electrical shocks have a potential to exacerbate $\mathrm{HF}$ via the mitochondoria-SR-Ca ${ }^{2+}$ positive 


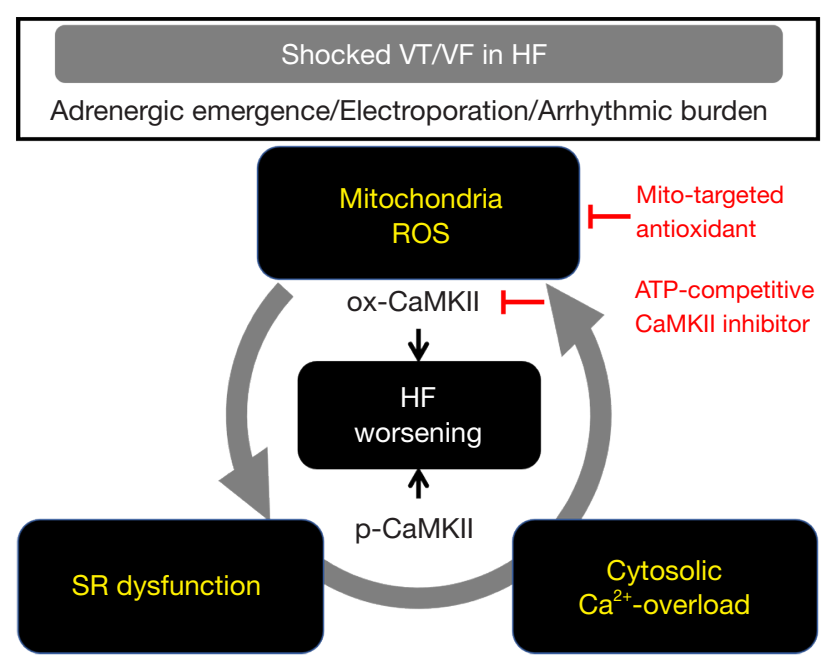

Figure 1 Proposed mechanism-based therapeutic approach for shocked VT/VF-associated HF. See details in text. VT/VF, ventricular tachycardia or fibrillation; HF, heart failure; CaMKII, $\mathrm{Ca}^{2+} /$ calmodulin-dependent protein kinase II; ox-CaMKII, oxidized form of CaMKII; p-CaMKII, auto-phosphorylated form of CaMKII; SR, sarcoplasmic reticulum; ROS, reactive oxygen species.

feedback loop. Shocked VT/VF-related elements, including electroporation, adrenergic emergence and arrhythmic burden, occur transiently, but may serve as a trigger for the loop acceleration. In an experimental model of ES created by inducing chronic complete atrioventricular (AV) block in ICD-implanted rabbits, which recapitulates the QT prolongation, the repetitive occurrence of Torsadesde-Pointes arrhythmias and the frequent induction VF episodes, we could show that VF storm causes $\mathrm{Ca}^{2+} /$ calmodulin-dependent protein kinase II (CaMKII) activation by increasing intracellular $\mathrm{Ca}^{2+}$ and promoting ROS generation (20,21). The ES-related fast activation rate, the chronic adrenergic stimulation and enhanced ROS generation cause persistent CaMKII activation (22). The auto-phosphorylated and oxidized forms ( $\mathrm{p}$ - and ox-CaMKII) with persistently-elevated enzyme activity phosphorylate multiple protein targets for excitationcontraction coupling, cell survival and transcription factors driving hypertrophic and inflammatory gene expression. The CaMKII-overactivity is connected to a variety of cardiac diseases including HF, myocardial infarction, ischemia/reperfusion and cardiac arrhythmias. Indeed, VF storm rabbits have striking upregulation of both p- and oxCaMKII, suggesting excess ROS production and elevated
$\mathrm{Ca}^{2+}$ levels $(20,21)$.

Mitochondria-targeted antioxidants and ATPcompetitive CaMKII inhibitors are highlighted as novel agents for HF. A mitochondria-targeted antioxidant (MitoTEMPO) prevents sudden cardiac death and reverses proteome remodeling by mitochondrial ROS scavenging in a guinea pig model of non-ischemic HF (23). Novel ATPcompetitive selective CaMKII inhibitors (GS-680 and AS105) improve contractility in human failing hearts and SR dysfunction in murine failing cardiomyocytes $(24,25)$. Future clinical trials assessing the efficacy of such treatment approaches for death prevention in ICD patients are needed to test and validate these putative therapeutic hypotheses.

\section{Acknowledgements}

Funding: The authors' work is supported by funds from Japan Society for the Promotion of Science (17K09511), the Suzuken Memorial Foundation, and APEX Co., Ltd. to Dr. Y Tsuji; by the National Institutes of Health (R01HL131517 and R01-HL136389) and the German Research Foundation (DFG, Do 769/4-1) to Dr. D Dobrev.

\section{Footnote}

Conflicts of Interest: The authors have no conflicts of interest to declare.

\section{References}

1. Chatzidou S, Kontogiannis C, Tsilimigras DI, et al. Propranolol versus metoprolol for treatment of electrical storm in patients with implantable cardioverterdefibrillator. J Am Coll Cardiol 2018;71:1897-906.

2. Chen PS, Doytchinova A. Why is propranolol better than metoprolol in acute treatment of electrical storm? J Am Coll Cardiol 2018;71:1907-9.

3. Tan VH, Wilton SB, Kuriachan V, et al. Impact of programming strategies aimed at reducing nonessential implantable cardioverter defibrillator therapies on mortality: A systematic review and meta-analysis. Circ Arrhythm Electrophysiol 2014;7:164-70.

4. Gasparini M, Proclemer A, Klersy C, et al. Effect of long-detection interval vs standard-detection interval for implantable cardioverter-defibrillators on antitachycardia pacing and shock delivery: The ADVANCE III randomized clinical trial. JAMA 2013;309:1903-11.

5. Sterns LD, Meine M, Kurita T, et al. Extended detection 
time to reduce shocks is safe in secondary prevention patients: The secondary prevention substudy of PainFree SST. Heart Rhythm 2016;13:1489-96.

6. Sapp JL, Wells GA, Parkash R, et al. Ventricular tachycardia ablation versus escalation of antiarrhythmic drugs. N Engl J Med 2016;375:111-21.

7. Santangeli P, Muser D, Maeda S, et al. Comparative effectiveness of antiarrhythmic drugs and catheter ablation for the prevention of recurrent ventricular tachycardia in patients with implantable cardioverter-defibrillators: A systematic review and meta-analysis of randomized controlled trials. Heart Rhythm 2016;13:1552-9.

8. Guerra F, Flori M, Bonelli P, et al. Electrical storm and heart failure worsening in implantable cardiac defibrillator patients. Europace 2015;17:247-54.

9. Nikolski VP, Efimov IR. Electroporation of the heart. Europace 2005;7 Suppl 2:146-54.

10. Wang Y'T, Efimov IR, Cheng Y. Electroporation induced by internal defibrillation shock with and without recovery in intact rabbit hearts. Am J Physiol Heart Circ Physiol 2012;303:H439-49.

11. Bode F, Wiegand U, Raasch W, et al. Differential effects of defibrillation on systemic and cardiac sympathetic activity. Heart 1998;79:560-7.

12. Cevik C, Perez-Verdia A, Nugent K. Implantable cardioverter defibrillators and their role in heart failure progression. Europace 2009;11:710-5.

13. Anderson ME. Calcium and stunning: When, from where, and what next? J Cardiovasc Electrophysiol 2002;13:1025-6.

14. Brewster J, Sexton T, Dhaliwal G, et al. Acute effects of implantable cardioverter-defibrillator shocks on biomarkers of myocardial injury, apoptosis, heart failure, and systemic inflammation. Pacing Clin Electrophysiol 2017;40:344-52.

15. Schirmer U, Hemmer W, Lindner KH, et al. Ultrastructural alterations in the right and left ventricular myocardium following multiple low energy endocardial

Cite this article as: Tsuji Y, Dobrev D. Electrical storm: mechanistic and therapeutic considerations to avoid death in the survivors. J Thorac Dis 2018;10(Suppl 33):S4053-S4056. doi: $10.21037 /$ jtd.2018.09.111 countershocks in anesthetized dogs. Pacing Clin Electrophysiol 1997;20:79-87.

16. Tsai MS, Huang CH, Tsai SH, et al. The difference in myocardial injuries and mitochondrial damages between asphyxial and ventricular fibrillation cardiac arrests. Am J Emerg Med 2012;30:1540-8.

17. Jones DL, Narayanan N. Defibrillation depresses heart sarcoplasmic reticulum calcium pump: a mechanism of postshock dysfunction. Am J Physiol 1998;274:H98-105.

18. Wagner S, Rokita AG, Anderson ME, et al. Redox regulation of sodium and calcium handling. Antioxid Redox Signal 2013;18:1063-77.

19. Dietl A, Maack C. Targeting mitochondrial calcium handling and reactive oxygen species in heart failure. Curr Heart Fail Rep 2017;14:338-49.

20. Tsuji $\mathrm{Y}$, Hojo $\mathrm{M}$, Voigt $\mathrm{N}$, et al. $\mathrm{Ca}^{2+}$-related signaling and protein phosphorylation abnormalities play central roles in a new experimental model of electrical storm. Circulation 2011;123:2192-203.

21. Yamazaki M, Tsuji Y, Niwa R, et al. Torsadogenic action of late $\mathrm{Na}^{+}$current in experimental electrical storm. Heart Rhythm 2018;15:S190-1.

22. Grandi E, Dobrev D. Non-ion channel therapeutics for heart failure and atrial fibrillation: Are CaMKII inhibitors ready for clinical use? J Mol Cell Cardiol 2018;121:300-3.

23. Dey S, DeMazumder D, Sidor A, et al. Mitochondrial ROS drive sudden cardiac death and chronic proteome remodeling in heart failure. Circ Res 2018;123:356-71.

24. Lebek S, Plossl A, Baier M, et al. The novel CaMKII inhibitor GS-680 reduces diastolic SR Ca leak and prevents CaMKII-dependent pro-arrhythmic activity. J Mol Cell Cardiol 2018;118:159-68.

25. Neef S, Steffens A, Pellicena P, et al. Improvement of cardiomyocyte function by a novel pyrimidine-based CaMKII-inhibitor. J Mol Cell Cardiol 2018;115:73-81. 